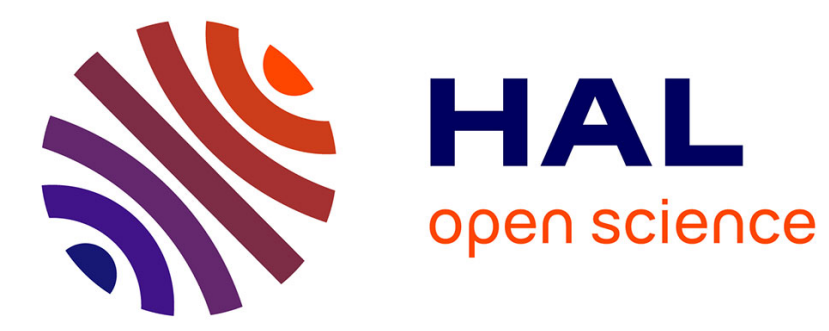

\title{
A new flight guidance approach based on differential flatness
}

\author{
Wen Chi Lu, Felix Mora-Camino, Karim Achaibou
}

\section{To cite this version:}

Wen Chi Lu, Felix Mora-Camino, Karim Achaibou. A new flight guidance approach based on differential flatness. DASC 2004, 23rd Digital Avionics System Conference, Oct 2004, Salt Lake City, United States. pp xxxx, 10.1109/DASC.2004.1390737 . hal-01021727

\section{HAL Id: hal-01021727 https://hal-enac.archives-ouvertes.fr/hal-01021727}

Submitted on 17 Jul 2014

HAL is a multi-disciplinary open access archive for the deposit and dissemination of scientific research documents, whether they are published or not. The documents may come from teaching and research institutions in France or abroad, or from public or private research centers.
L'archive ouverte pluridisciplinaire HAL, est destinée au dépôt et à la diffusion de documents scientifiques de niveau recherche, publiés ou non, émanant des établissements d'enseignement et de recherche français ou étrangers, des laboratoires publics ou privés. 


\title{
A NEW FLIGHT GUIDANCE APPROACH BASED ON DIFFERENTIAL FLATNESS
}

\author{
Wen-Chi Lu, LAAS du CNRS and ENAC/UT2/MARTA, Toulouse, France \\ Félix Mora-Camino, LAAS du CNRS and ENAC, Toulouse, France \\ Karim Achaibou, LAAS du CNRS, Toulouse, France
}

\begin{abstract}
With the sustained increase of air traffic, the demand for new maneuvering capabilities of transport aircrafts are getting more importance in order to tackle traffic saturated conditions. In this communication, a new guidance control structure is proposed to perform trajectory tracking through smooth manoeuvers. The design approach is based first on a differential flatness property of aircraft guidance dynamics which is displayed in this paper and then on the use of neural networks to invert the guidance dynamics in order to generate directives to the autopilot. To compensate the effect of modeling errors and of other disturbances, an adaptive control approach is adopted to perform the maneuvers.
\end{abstract}

\section{Introduction}

The sustained increase of air traffic in the airports of most regional hubs and big cities worldwide inevitablely leads to traffic saturated situations. To alleviate the traffic load and to solve saturated problems in these airports, except for the improvement on air traffic management system (ATM), the development of new maneuvering capabilities for transport aircrafts is also one of the solutions.

Recent progress in differential geometry has led a new branch of the control theories of nonlinear systems. Their applicability to flight dynamics which are essentially nonlinear, has been of utmost interest while previous flight control functions were mainly based on cumbersome gainscheduling linear controllers. Differential flatness, a concept introduced by Fliess et al. [1] from the theory of differential geometry, has provided new opportunities to design advanced control schemes for nonlinear systems. For a flat system, given a trajectory for a flat output, it becomes easy to derive the corresponding input. A number of articles have already given some insight into the differential flatness of aircraft flight dynamics [2], but none of them has considered separately the flatness property of the flight guidance dynamics for a rigid aircraft.

In this communication, a new guidance control structure is proposed to perform trajectory tracking through smooth manoeuvers. The design approach is based on the differential flatness property of aircraft guidance dynamics which is displayed in this paper and on the use of feedforward neural networks to invert the guidance dynamics and then to generate basic directives to the autopilot. In order to compensate the effect of modeling errors and of external disturbances, an adaptive control approach is proposed to perform the maneuvers.

\section{Differential Flatness and Nonlinear Systems}

Here two definitions of flatness are introduced: - one relative to systems for which causal relationships are displayed analytically, it is explicit flatness.

- another one, implicit flatness, where the causal relationships are introduced through implicit functions.

Note, that the term differential is dropped in the remaining of this paper to avoid cumbersome denominations.

Definition 1: A general nonlinear system given by:

$$
\underline{\dot{X}}=f(\underline{X}, \underline{U}), \quad \underline{X} \in R^{n}, \underline{U} \in R^{m}
$$

where $\mathrm{f}$ is a smooth mapping, is said explicitly flat with respect to the output vector $\underline{Z}$, if $\underline{Z}$ is an $m^{\text {th }}$ order vector which can be expressed analytically as a function of the current state, the current input and its derivatives and also such as the state and the input vectors can be expressed analytically as a function of $\underline{Z}$ and its derivatives. Then there exists smooth mappings $F_{X}, F_{U}$, and $F_{Z}$ such as: 


$$
\begin{aligned}
& \underline{Z}=F_{Z}\left(\underline{X}, \underline{U}, \ldots, \underline{U}^{(p)}\right) \\
& \underline{X}=F_{X}\left(\underline{Z}, \underline{\dot{Z}}, \ldots, \underline{Z}^{(q)}\right) \\
& \underline{U}=F_{U}\left(\underline{Z}, \underline{Z}, \ldots, \underline{Z}^{(q+1)}\right)
\end{aligned}
$$

where $p$ and $q$ are integer numbers.

Vector $\underline{Z}$ is called a flat output for nonlinear system (1).

Observe that when a flat output exists, it is not unique. The explicit flatness property is of particular interest for the solution of a control problem when a physically meaningful flat output can be related with its objectives. In many situations, the control problem can be formulated as a flat output trajectory following problem. In general, for these cases, the flat output of (2a) can be reduced, through state transformation, to a function of a single argument, the new system state itself:

$$
\underline{Z}=F_{z}(\underline{X})
$$

However, for many systems, no complete analytical models are available to describe their full dynamics. Then some of their components make use of input-output numerical devices derived both from theory and from experimental data. In these cases, available theory provides in general the main mathematical properties of these implicit functions while experimental data is used to build accurate input output numerical devices. This happens for instance when flight dynamics modeling is considered either for control or simulation purposes, since many often, the aerodynamic coefficients are obtained from a set of look up discrete numerical tables and through complex interpolation computing.

Definition 2: A nonlinear system given by a general implicit $\mathrm{n}^{\text {th }}$ order state representation:

$$
F(\underline{X}, \underline{\dot{X}}, \underline{U})=0 \quad \underline{X} \in R^{n}, \underline{U} \in R^{m}
$$

where $F$ is a regular implicit mapping with respect to $\underline{\dot{X}}$, is said implicitly flat over an interior non empty domain $\Delta \subseteq \mathrm{R}^{\mathrm{n}+\mathrm{m}}$ if it is possible to find a $\mathrm{m}^{\text {th }}$ order vector $\underline{Z}$ which obeys conditions (2) and condition:

$$
G\left(\underline{X}, \underline{U}, \underline{Z}, \underline{\dot{z}}, \ldots, \underline{Z}^{(r)}\right)=\underline{0}
$$

where $G$ is locally invertible over $\Delta$ with respect to $\underline{X}$ and $\underline{U}$ where $r$ is an integer number.

Again, vector $\underline{Z}$ is said to be a flat output. The invertibility of $G$ is guarranted if the determinant of the Jacobian of $G$ is not zero according to the theorem of implicit functions, i.e. if:

$$
\operatorname{det}\left(\frac{\partial G}{\partial(\underline{X}, \underline{U})}\right) \neq 0 \quad \text { over } \Delta
$$

The relation (4) and the condition (5) show that given a trajectory for the flat output $\underline{Z}$, it is still possible to map it numerically into the input space to get an adequate control law so that one of the more helpful properties of explicitly flat systems is still maintained. Furthermore, any established property for implicitly flat systems will also hold in numerical ground for explicitly flat systems since they can straightforwardly satisfy relation (6).

\section{Flight Guidance Dynamics}

In this study, the guidance dynamics of transportation aircraft are only considered since it is assumed that the aircraft of interest are equiped with a basic auto-pilot which deals efficiently with their fast dynamics and thus controls their body attitude $(\theta, \phi, \beta)$ and the $N_{1}$ regime of their engines. It is also assumed that turn manoeuvers are achieved in a coordinated way ( $\beta$ remaining approximately null). Then, supposing also a no wind condition and making use of the aerodynamic reference frame, the flight guidance equations can be written:

$$
\begin{gathered}
V_{x}=V \sin \psi \cos \gamma \\
V_{y}=V \cos \psi \cos \gamma \\
V_{z}=-V \sin \gamma \\
\text { with } V=\sqrt{V_{x}^{2}+V_{y}^{2}+V_{z}^{2}} \\
\dot{V}=\frac{-D+T \cos \alpha-m g(-\cos \alpha \sin \theta+\sin \alpha \cos \phi \cos \theta)}{m}
\end{gathered}
$$


$\dot{\gamma}=\frac{L \cos \phi+T \sin \alpha-m g(\sin \alpha \sin \theta+\cos \alpha \cos \phi \cos \theta)}{m V}$

(7e)

When perfect coordinated turns are achieved, the heading rate is related to the bank-angle $\phi$ through the following relation:

$$
\dot{\psi}=(g / V) \tan (\phi) \cos (\theta)
$$

The drag $D$ and lift $L$ are considered to be respectively smooth functions of altitude $z$, airspeed $V$ and angle-of-attack $\alpha$. While the thrust $T$ of a turbofan engines is considered to be a smooth function of altitude $z$, airspeed $V$ and of their common fan regime $N_{1}$. Then drag, lift and thrust are such as:

$$
\begin{aligned}
& D=D(z, V, \alpha) \\
& L=L(z, V, \alpha) \\
& T=T\left(z, V, N_{1}\right)
\end{aligned}
$$

For local guidance purposes, the flight-path angle $\gamma$ becomes the control parameter and the angle-of-attack $\alpha$ can be expressed in terms of $\gamma, \theta$ and $\phi$. When the bank angle is very small, this relation can be written:

$$
\alpha=\theta-\gamma
$$

It appears from the above equations that the flight variables $\theta, \phi$ and $N_{1}$ can be taken as the inputs for the guidance dynamics while they are output variables for the body frame dynamics when controlled by a basic auto pilot. Figure 1 displays the resulting structure for the whole flight dynamics:

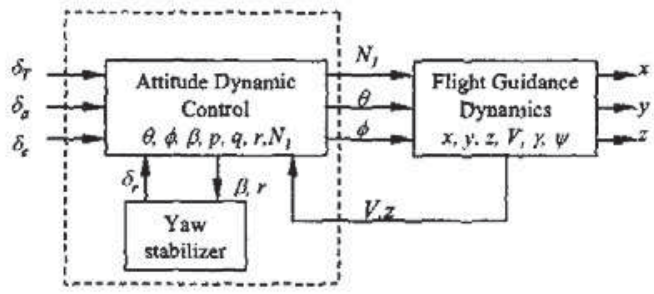

Figure 1. Aircraft Flight Dynamics Structure

\section{Flight Guidance Dynamics and Differential Flatness}

By rearranging the kinematical equations ( $7 \mathrm{a}$, $7 \mathrm{~b}$, and $7 \mathrm{c})$, it is convenient to express $V, \gamma$ and $\psi$ as:

$$
\begin{aligned}
& V=\left((\dot{x})^{2}+(\dot{y})^{2}+(\dot{z})^{2}\right)^{\frac{1}{2}} \\
& \gamma=-\sin ^{-1}(\dot{z} / V) \\
& \psi=\tan ^{-1}(\dot{y} / \dot{x})
\end{aligned}
$$

The state variables obviously can be functions of the position of the aircraft while the control variables satisfy the following relations:

$$
\begin{gathered}
\dot{V}-\frac{-D+T \cos \alpha-m g(-\cos \alpha \sin \theta+\sin \alpha \cos \phi \cos \theta)}{m}=0 \\
\dot{\gamma}+\frac{-L \cos \phi-T \sin \alpha+m g(\sin \alpha \sin \theta+\cos \alpha) \cos \phi \cos \theta)}{m V}=0 \\
\dot{\psi}-(g / V) \operatorname{tg} \phi \cos \theta=0
\end{gathered}
$$

where $\alpha$ is given by relation (9).

The following notations are adopted here for the position coordinates of the center of gravity of the aircraft:

$$
\underline{Z}=(x, y, z)^{T}
$$

And for the guidance inputs:

$$
\underline{U}=\left(\theta, \phi, N_{1}\right)
$$

When, and it is very often the case, no analytical expressions are available for the forces exerted on the aircraft, the equations (12a), (12b) and $(12 c)$ with (9a) can be regarded as implicit functions of the position vector $\underline{Z}$, of its first two derivatives with respect to time and of input $\underline{U}$.They can be rewritten as:

$$
\begin{aligned}
& G_{N_{1}}(\underline{Z}, \underline{\dot{Z}}, \underline{Z}, \underline{U})=0 \\
& G_{\theta}(\underline{Z}, \underline{Z}, \underline{Z}, \underline{U})=0 \\
& G_{\phi}(\underline{Z}, \underline{\dot{Z}}, \underline{Z}, \underline{U})=0
\end{aligned}
$$

These implicit functions are locally invertible with respect to the input since for normal flight conditions the determinant of their Jacobian is not zero: 


$$
\left|\begin{array}{lll}
\frac{\partial G_{N_{1}}}{\partial \theta} & \frac{\partial G_{N_{1}}}{\partial \phi} & \frac{\partial G_{N_{1}}}{\partial N_{1}} \\
\frac{\partial G_{\theta}}{\partial \theta} & \frac{\partial G_{\theta}}{\partial \phi} & \frac{\partial G_{\theta}}{\partial N_{1}} \\
\frac{\partial G_{\phi}}{\partial \theta} & \frac{\partial G_{\phi}}{\partial \phi} & \frac{\partial G_{\phi}}{\partial N_{1}}
\end{array}\right| \neq 0
$$

Then, the considered flight guidance dynamics are implicit flat with $\underline{Z}=(x, y, z)^{T}$ as their flat output vector. The time evolution of these flat outputs represents the trajectory followed by the center of gravity of the aircraft. Then according to the above theory, from the knowledge of this trajectory, it is possible to find the corresponding input trajectory.

\section{Autopilot Guidance Reference Generation} output:

Given a smooth reference trajectory for the flat

$$
\underline{Z}_{c}(\tau)=\left(\mathrm{x}_{c}(\tau), \mathrm{y}_{c}(\tau), \mathrm{z}_{\mathrm{c}}(\tau)\right)^{T}, \tau \in\left[\mathrm{t}_{0}, \mathrm{t}\right]
$$

the corresponding reference input values at time $t$, $\underline{U}_{c}(\mathrm{t})=\left(\theta_{c}(\mathrm{t}), \phi_{c}(\mathrm{t}), N_{l c}(\mathrm{t})\right)^{T}$, are the solutions of the equations:

$$
\begin{aligned}
& G_{N_{1}}\left(\underline{Z}_{c}(t), \underline{\dot{Z}}_{c}(t), \underline{\underline{Z}}_{c}(t), \underline{U}_{c}(t)\right)=0 \\
& G_{\theta}\left(\underline{Z}_{c}(t), \underline{\dot{Z}}_{c}(t), \ddot{Z}_{c}(t), \underline{U}_{c}(t)\right)=0 \\
& G_{\phi}\left(\underline{Z}_{c}(t), \underline{\dot{Z}}_{c}(t), \underline{Z}_{c}(t), \underline{U}_{c}(t)\right)=0
\end{aligned}
$$

where $\underline{\underline{Z}}_{c}(t), \underline{\underline{Z}}_{c}(t)$ and $\underline{\underline{z}}_{c}(t)$ are the current parameters.

Since it is not possible to get an on line numerical solution to this set of implicit equations, a neural network can be designed beforehand to build the above input/output mappings where the current parameters are the inputs and the components of the control vector are the outputs (see Figure 2). Then these input/output mappings will be available for on line operation.

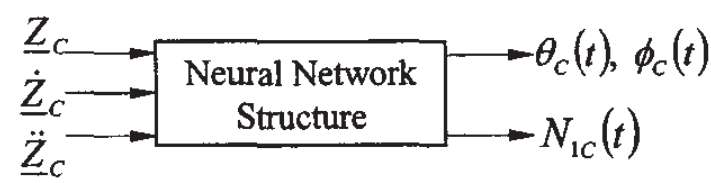

Figure 2. Reference Input Generator by NeuralNetworks

A multilayer neural network can be trained through the minimization of a generalized mean square error between field trajectory data and computed outputs.

$$
\min _{W} \sum_{i \in l} \sum_{k \in K_{i}}\left\|\underline{U}_{N N}\left(W, \underline{Z}\left(t_{i k}\right), \underline{Z}\left(t_{i k}\right), \underline{Z}\left(t_{i k}\right)\right)-\underline{U}\left(t_{i k}\right)\right\|_{Q}^{2}
$$

where $I$ is the set of training trajectories, $K_{i}$ is the set of training points of the $i^{\text {th }}$ trajectory, $W$ is the matrix of the weights of the neural network. $\underline{U}_{N N}$ is the neural network computed value for the input $\underline{U}$.

Here matrix $Q$ can be chosen as:

$$
Q=\operatorname{diag}\left\{1 / N_{I_{\max }}, 1 / \theta_{\max }, 1 / \phi_{\max }\right\}
$$

Many design and minimization tools are available to perform this task [3] and to achieve acceptable accuracy levels as long as sufficient training data is at hand. Here the training data is composed of sets of trajectories for $\underline{Z}$ and $\underline{U}$ which can be provided from either flight test data or even from commercial flight data where maneuvers are manually performed or when the auto pilot is operating under basic modes (no guidance loop must be active at that time). Observe here that modern aircraft onboard navigation systems (ADIRS) are able to estimate with good accuracy the current aircraft position, inertial speed and wind speed and record them, so that preprocessing of the training data may be minimized using available flight records.

\section{A Neural Flight Guidance Control}

The Figure 3 displays a possible flight guidance control structure which integrates a neural network solver. Tactical manoeuvres can be generated from the confrontation of the actual position and speed of the aircraft with its flight plan and from the intervention of the pilot through his control display unit (CDU). The pilot, through the audio channel and the cockpit display traffic information (CDTI) can receive tactical information 
from the air traffic control system. Then the reference trajectory can be generated from the actual position and speed of the aircraft and the tactical manoeuvre under way. Observe that if it can be useful to the pilot to get a graphical representation of the reference trajectory on the navigation display (ND), the neural network solver, like a flight director, needs only the current position and tendency to the second order. To provide smooth reference values to the guidance system, these outputs can also be post processed by linear filters and also checked by a flight envelope control logic. Since the navigation loop is used with a discrete time scale, the drift resulting from modeling errors and perturbations will be compensated by introducing at each current sampled time an updated reference trajectory. This approach has already been proposed in [4]. In the case of a non standard approach manoeuvre, simplified adaptive reference trajectories such as:

$$
\begin{aligned}
& x_{c}(\tau)=x(t)+\int_{t}^{\tau}\left\{\dot{x}(t) e^{-s / T_{x}}+V_{0} \cos \gamma_{0}\left(1-e^{-s / T_{X}}\right\} d s\right. \\
& y_{c}(\tau)=y(t)+\dot{y}(t)(\tau-t) e^{-(\tau-t)} \\
& z_{c}(\tau)=z(t)+\int_{i}^{\tau}\left\{\dot{z}(t) e^{-s / T_{z}}+V_{0} \sin \gamma_{0}\left(1-e^{-s / T_{2}}\right\} d s\right.
\end{aligned}
$$

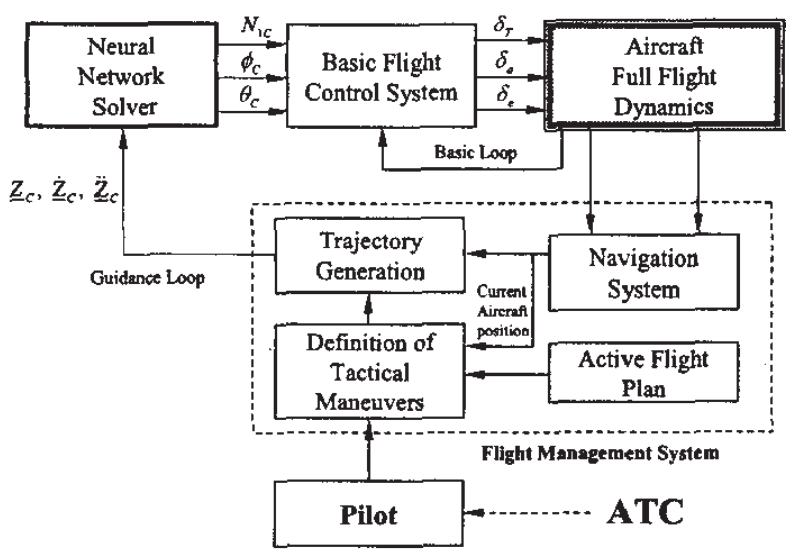

Figure 3. A Possible Flight Guidance Control Structure

can be adopted, where $T_{X}, T_{Y}$ and $T_{Z}$ are time constants which provide a time reference for the transition between current conditions and final guidance goals (here $V_{\infty}=V_{0} \cos \gamma_{0}, y_{\infty}=0$ and $v_{z \infty}$ $=\mathrm{V}_{0} \sin \gamma_{0}$ ). Here since the innovative effect appears mainly with the second time derivative of the position, the generated aircraft trajectories should be particularly smooth. Otherwise, when hard final constraints are pursued, a polynomial construction of the reference trajectory will be of interest.

\section{Simulation Results}

Some preliminary simulation results are displayed in Figures 4 to 8 in order to validate the proposed approach. Based on the public released dynamic model of a general aviation aircraft, North American Navion Aircraft [5, 6], a neural network structure has been built for controller synthesis. It is composed of two neural networks, one is devoted to the generation of pitch and bank angles references while another provides a reference value for the engine regime. Figures 4 and 7 display some information about their training history, while Figures 5, 6 and 8 provide an assessment of the accuracy and of the power of generalization of these trained neural networks. It appears that the training of these neural networks is quite easy; this may be explained by the strength of the flatness property present in the flight dynamic equations.

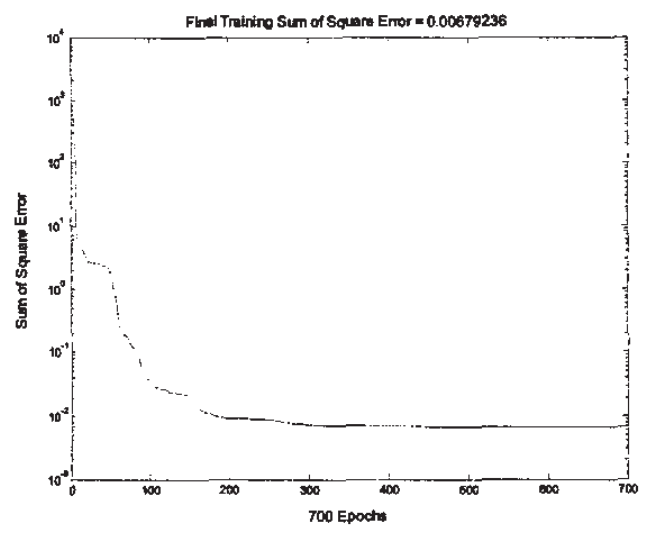

Figure 4. Sum of Square-Errors in Training Process for Bank Angle and Pitch Angle 

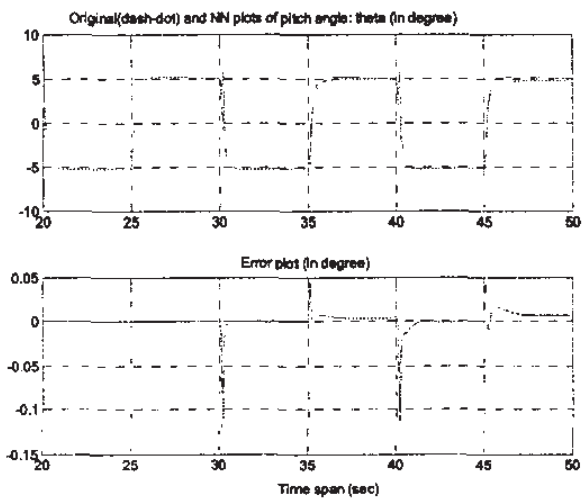

Figure 5. Validation Result of Generalization for Bank Angle

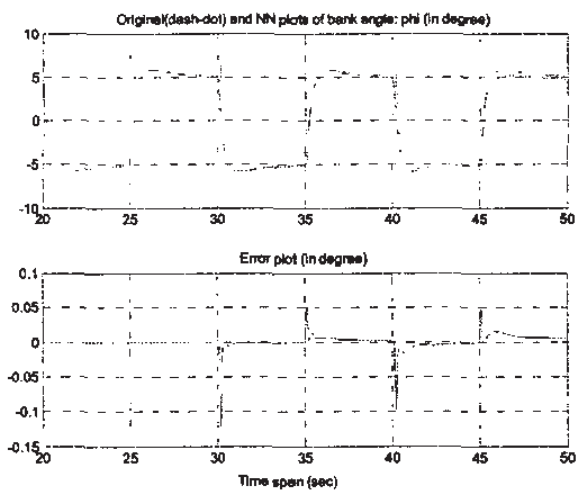

Figure 6. Validation Result of Generalization for Pitch Angle

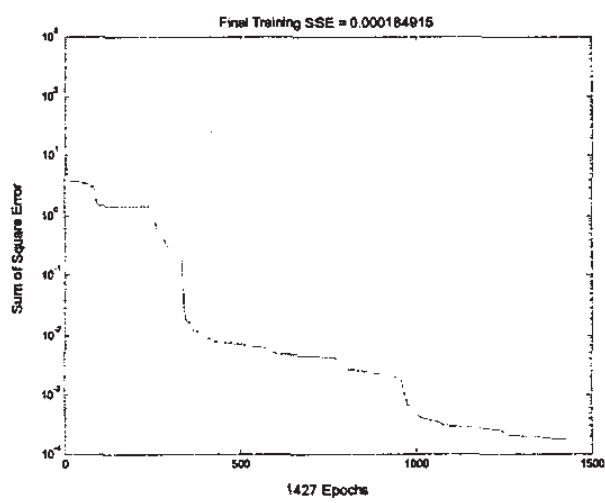

Figure 7. Sum of Square-Errors in Training Process for Engine Regime N1
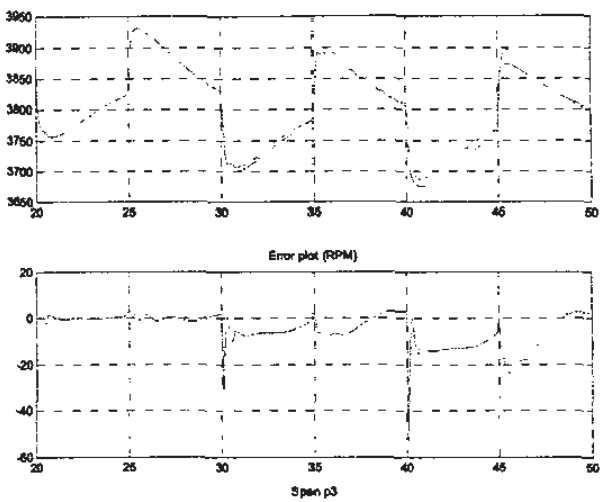

Figure 8. Validation Result of Generalization for Engine Regime N1

\section{Conclusion}

This paper opens the way to important new developments in the field of flight guidance control law design, as well as in the field of flight guidance systems technology:

It provides a generic guidance system compatible with basic autopilot systems and allows to perform the tracking of many different trajectories, either classical or not.

Differential flatness theory provides a sound basis for the introduction of neural networks technology in flight guidance systems.

Then design cost for guidance functions should be substantially reduced while improved guidance performances should be obtained more easily.

\section{References}

[1] M. Fliess, J. Lévine, P. Martin, and P. Rouchon, 1995, Flatness and defect of non-linear systems: theory and examples, Int. J. Control, , Vol. 61, No. 6, pp. 1327-1361.

[2] L.Lavigne, F. Cazaurang, B. Bergeon, 2003, Modelling of Longitudinal Disturbed Aircraft Model by Flatness Approach, AIAA Guidance, Navigation, and Control Conference and Exhibit, Texas Austin, USA.

[3] Neural Network Toolbox for Use with

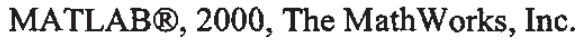


[4] F. Mora-Camino, A. Achaibou, T. Miquel, R. Faye, B. Ouattara and S. Sawadogo, 2004, Minimum time trajectory generation for relative guidance of aircraft, IPDO-Inverse Problems, Design and Optimisation symposium, Rio de Janeiro, Brazil.

[5] AEROSIM BLOCKSET User's Guide, Unmanned Dynamics, LLC.

[6] Louis V. Schmidt, Introduction to Aircraft Flight Dynamics, ALAA Education series, 1998.

\section{Bibliography}

[7] R.C. Nelson, 1989, Flight Stability and Automatic Control, McGraw-Hill.

[8] Brian L. Steven, Frank L. Lewis, 1992, Aircraft Control and Simulation, John Wiley \& Sons, Inc.

[9] W. Lu, F. Mora-Camino, M. de Coligny, F. Lavergne, K. Achaibou, 2004, Flight Mechanics and Differential Flatness, DINCON 2004, Ilha Solteira, Brazil, pp. 830-839.
[10] W. Lu, F. Mora-Camino, 2004, Systemes Plats et La Dynamique du Vol, EDSYS Congrès Doctorant 2004, Toulouse, France.

\section{Nomenclature}

$\underline{X} \underline{U} \underline{Z}$ : state, control input and flat output vectors, $x, y, z$ : position of aircraft in the local Earth frame, $F, F_{i}, G, G_{j}$ : multidimensional mappings, $V$ : aircraft speed, $v_{y}:$ lateral aircraft speed, $\alpha$ : angle-of-attack, $\beta$ : side-slip angle and $\gamma$ air vertical path angle,

$\theta, \phi, \psi \cdot$ pitch, bank and heading angles,

$p, q, r$ : roll, pitch and yaw angular rates,

$\delta_{e}, \delta_{a}, \delta_{r}, \delta_{T}$ : elevator, aileron, rudder and engine settings,

$L, D, T$ : lift, drag and thrust forces,

$N_{l}$ : fan regime,

$m$ : aircraft mass, $g$ : gravitational acceleration 